\title{
ADIPOSE TISSUE-DERIVED MICROVASCULAR FRAGMENTS FROM AGED DONORS EXHIBIT AN IMPAIRED VASCULARISATION CAPACITY
}

\author{
M.W. Laschke ${ }^{1,2, *}$, C. Grässer ${ }^{1,2}$, S. Kleer ${ }^{1,2}$, C. Scheuer ${ }^{1,2}$, D. Eglin ${ }^{3}$, M. Alini ${ }^{3}$ and M.D. Menger ${ }^{1,2}$ \\ ${ }^{1}$ Institute for Clinical and Experimental Surgery, University of Saarland, 66421 Homburg/Saar, Germany \\ ${ }^{2}$ Collaborative Research Partner Large Bone Defect Healing Program of AO Foundation \\ ${ }^{3}$ AO Research Institute Davos, Clavadelerstrasse, 7270 Davos Platz, Switzerland
}

\begin{abstract}
Adipose tissue-derived microvascular fragments are promising vascularisation units for applications in the field of tissue engineering. Elderly patients are the major future target population of such applications due to an increasing human life expectancy. Therefore, we herein investigated the effect of aging on the fragments' vascularisation capacity. Microvascular fragments were isolated from epididymal fat pads of adult ( 8 months) and aged (16 months) C57BL/6 donor mice. These fragments were seeded onto porous polyurethane scaffolds, which were implanted into dorsal skinfold chambers to study their vascularisation using intravital fluorescence microscopy, histology and immunohistochemistry. Scaffolds seeded with fragments from aged donors exhibited a significantly lower functional microvessel density and intravascular blood flow velocity. This was associated with an impaired vessel maturation, as indicated by vessel wall irregularities, constantly elevated diameters and a lower fraction of CD31/ $\alpha$-smooth muscle actin double positive microvessels in the implants' border and centre zones. Additional in vitro analyses revealed that microvascular fragments from adult and aged donors do not differ in their stem cell content as well as in their release of angiogenic growth factors, survival and proliferative activity under hypoxic conditions. However, fragments from aged donors exhibit a significantly lower number of matrix metalloproteinase -9-positive perivascular cells. Taken together, these findings demonstrate that aging is a crucial determinant for the vascularisation capacity of isolated microvascular fragments.
\end{abstract}

Keywords: Tissue engineering, microvascular fragments, aging, senescence, angiogenesis, scaffold, polyurethane, dorsal skinfold chamber, intravital fluorescence microscopy.

\author{
*Address for correspondence: \\ Matthias W. Laschke \\ Institute for Clinical and Experimental Surgery \\ University of Saarland \\ D-66421 Homburg/Saar \\ Germany
}

Telephone Number: +496841 1626554

FAX Number: +496841 1626553

E-mail: matthias.laschke@uks.eu

\section{Introduction}

Survival and long-term function of tissue constructs are crucially dependent on the rapid establishment of a sufficient blood supply after their implantation into a defect site (Laschke et al., 2006a; Novosel et al., 2011). This, however, cannot be solely achieved by the ingrowth of blood vessels from the surrounding tissue, because the physiological growth rate of newly developing microvessels is estimated not to be faster than $\sim 5 \mu \mathrm{m} / \mathrm{h}$ (Zarem, 1969; Orr et al., 2003). To overcome this problem, sophisticated prevascularisation strategies have emerged in the field of tissue engineering during the last years (Rivron et al., 2008; Muscari et al., 2014). All of these strategies are based on the common concept of generating preformed microvascular networks within tissue constructs prior to their implantation, which may be rapidly blood-perfused by developing interconnections to the host microvasculature at the implantation site (Laschke et al., 2009; Laschke and Menger, 2012).

As in the case of other cell-based therapeutic approaches (Stegemann et al., 2014), the clinical introduction of prevascularisation strategies faces significant technical, regulatory and policy hurdles. Hence, rather simple yet effective strategies may have the best chance to enter clinical practice in the medium term. In line with this point of view, we and others recently reported that adipose tissue-derived microvascular fragments are promising prevascularisation units for tissue engineering (Shepherd et al., 2007; Hiscox et al., 2008; Laschke et al., 2012; Pilia et al., 2014). Importantly, these mixed arteriolar, capillary and venular vessel segments can be easily isolated by enzymatic digestion of fat tissue (Hoying et al., 1996; Shepherd et al., 2004; Nunes et al., 2010). Moreover, they rapidly reassemble to functional microvascular networks within implanted tissue constructs (Laschke et al., 2012). This circumvents the need for a complex and time-consuming de novo formation of mature vessels in vitro or in situ, which requires the long-term cultivation of cell-seeded scaffolds or the implantation of empty scaffolds at well vascularised sites (Laschke et al., 2008; Duttenhoefer et al., 2013). Accordingly, in future clinical practice microvascular fragments may be harvested from a patient in large amounts by minimally-invasive liposuction and directly transferred to a defect site without any further manipulation in an intra-operative one-step procedure.

This approach, however, raises the important question, if there are relevant patient-associated differences in the quality of isolated microvascular fragments. It should be considered that the number of elderly patients will increase substantially during the next decades due to an increasing 
average human life expectancy. This makes them a major target population of future tissue engineering applications (Choudhery et al., 2014). Therefore, the aim of the present study was to analyse whether the vascularisation potential of microvascular fragments depends on the age of the fat sample donor.

For this purpose, we isolated microvascular fragments from epididymal adipose tissue of adult and aged C57BL/6 mice. These fragments were seeded onto porous polyurethane scaffolds, which were implanted into dorsal skinfold chambers of recipient mice to study their vascularisation by means of repetitive intravital fluorescence microscopy, histology, and immunohistochemistry. Additional in vitro experiments were performed, to assess the stem cell content of microvascular fragments derived from donors of different age as well as their survival, proliferating activity and release of angiogenic growth factors under hypoxic conditions.

\section{Materials and Methods}

\section{Animals}

All animal experiments were approved by the local governmental animal care committee and were conducted in accordance with the German legislation on protection of animals and the NIH Guidelines for the Care and Use of Laboratory Animals (NIH Publication \#85-23 Rev. 1985).

To analyse whether the vascularisation potential of microvascular fragments depends on the age of the fat sample donors, microvascular fragments were isolated from the epididymal fat pads of C57BL/6 mice (Institute for Clinical and Experimental Surgery, University of Saarland, Homburg/Saar, Germany) with an average age of 8 months (hereinafter referred to as adult donors) or 16 months (hereinafter referred to as aged donors) (Fig. 1a). Importantly, both groups of donor animals exhibited a body weight of $>30 \mathrm{~g}$ (Fig. 1b), which was necessary for harvesting a sufficient amount of epididymal adipose tissue for the isolation of microvascular fragments.

For the preparation of dorsal skinfold chambers, we used C57BL/6 mice with an average age of 3-4 months and a body weight of 22-26 g. The animals were housed one per cage and had free access to tap water and standard pellet food (Altromin, Lage, Germany).

\section{Isolation of microvascular fragments and seeding of scaffolds}

The donor mice were anaesthetised by intraperitoneal (i.p.) injection of ketamine $(75 \mathrm{mg} / \mathrm{kg}$ body weight; Pharmacia, Erlangen, Germany) and xylazine $(25 \mathrm{mg} / \mathrm{kg}$ body weight; Rompun, Bayer, Leverkusen, Germany). After midline laparotomy, the epididymal fat pads were harvested (Fig. 1c) and enzymatically digested for the isolation of microvascular fragments (Fig. 1d), as described previously in detail (Laschke et al., 2012).

Directly after the isolation procedure, the microvascular fragments were seeded on porous nano-size hydroxyapatite particles/poly(ester-urethane) composite scaffolds with a size of $\sim 3 \times 3 \times 1 \mathrm{~mm}$ and interconnected macropores of
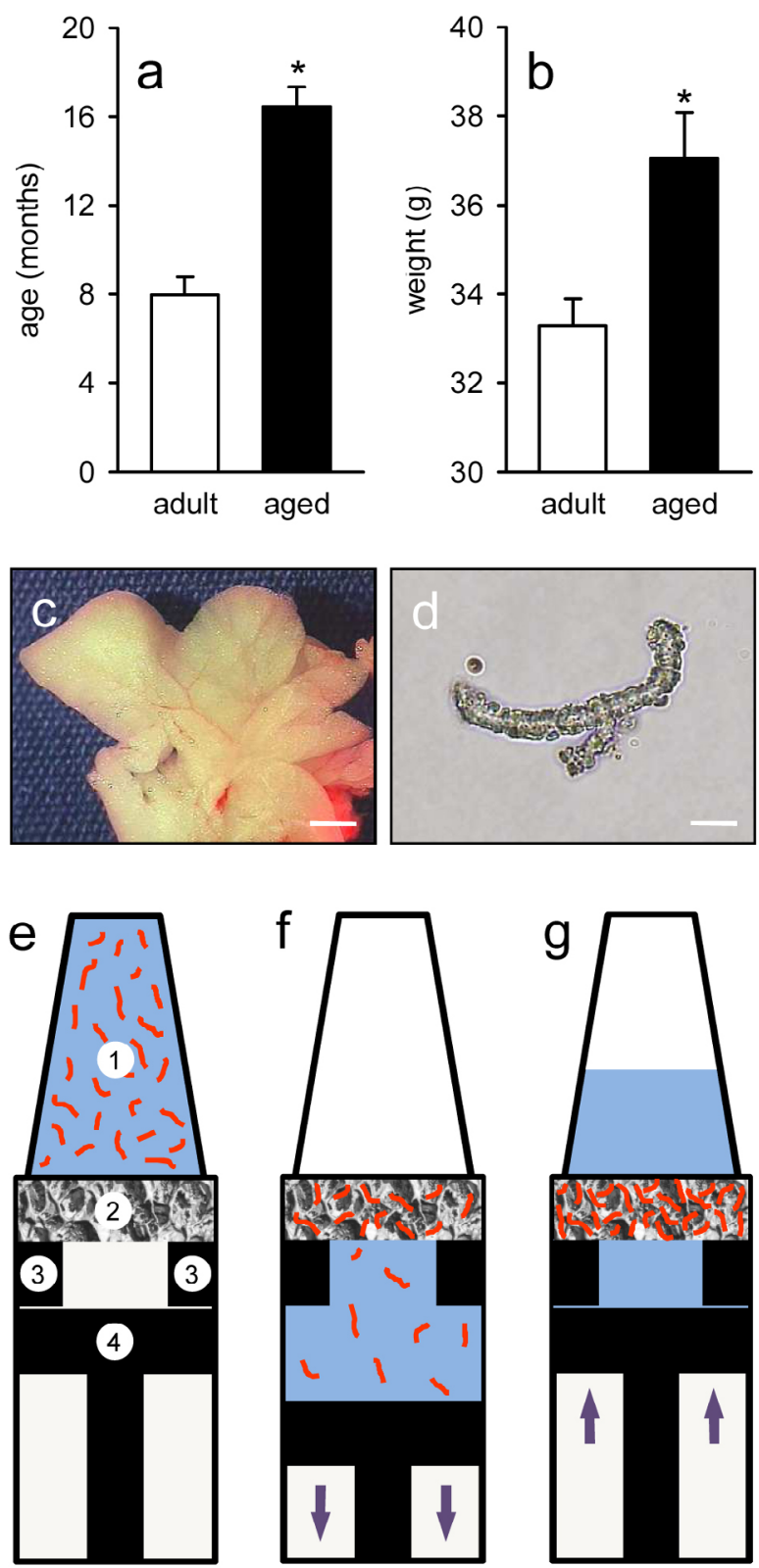

Fig. 1. (a, b): Age (months) (a) and body weight (g) (b) of adult (white bars, $n=8$ ) and aged (black bars, $n=8$ ) C57BL/6 mice, which served as donor animals for the isolation of microvascular fragments from epididymal fat pads. Means \pm SEM. ${ }^{*} p<0.05 v s$. adult donors. (c) Vessel-containing epididymal fat pad harvested from an adult donor mouse. (d) Microscopic image of a microvascular fragment directly after the isolation from the fat pad. Scale bars: $(\mathbf{c})=2 \mathrm{~mm} ;(\mathbf{d})=30 \mu \mathrm{m}$. (e-g): Dynamic seeding of a porous polyurethane scaffold with microvascular fragments. The scaffold $(\mathbf{e}, \mathbf{2})$ is fixed in the lumen of a modified $1 \mathrm{~mL}$ syringe by means of a rubber ring (e, 3$)$. The tip of the syringe is filled with $20 \mu \mathrm{L}$ PBS containing the microvascular fragments (e, 1). Negative (f) and positive pressure (g) is alternately induced in the syringe until all microvascular fragments are trapped in the scaffold pores. 
$\sim 220 \mu \mathrm{m}$ (Laschke et al., 2010a). These elastic scaffolds exhibit a good in vivo biocompatibility and, thus, have been used in several former studies as standardised matrices for the testing of novel vascularisation strategies for tissue engineering (Laschke et al., 2010b; Laschke et al., 2011a; Laschke et al., 2013). For the dynamic seeding procedure, the scaffolds were fixed in the lumen of a modified $1 \mathrm{~mL}$ syringe (BD Plastipak; BD Biosciences, Heidelberg, Germany). The tip of the syringe was filled with $20 \mu \mathrm{L}$ phosphate-buffered saline (PBS) containing the microvascular fragments (Fig. 1e). Subsequently, negative and positive pressure was alternately induced three to five times in the syringe so that the microvascular fragments could pass the scaffolds from both sides and were finally trapped in the scaffold pores (Figs. 1f,g).

\section{Dorsal skinfold chamber model and intravital fluorescence microscopy}

The vascularisation of seeded scaffolds was analysed in the dorsal skinfold chamber of C57BL/6 recipient mice by means of repetitive intravital fluorescence microscopy (Fig. 2a). A detailed description of the chamber preparation is given by Laschke et al. (2011b). After the surgical procedure, the mice were allowed to recover for $48 \mathrm{~h}$. Then, the cover glass of the dorsal skinfold chamber was temporarily removed and one scaffold seeded with microvascular fragments was placed onto the striated muscle tissue within the centre of each chamber (Fig. 2b).

For intravital fluorescence microscopy, $0.05 \mathrm{~mL}$ $5 \%$ fluorescein isothiocyanate (FITC)-labelled dextran 150,000 (Sigma-Aldrich, Taufkirchen, Germany) was injected intravenously (i.v.) via the retrobulbar space of the anaesthetised mice. This provided adequate contrast enhancement by staining of blood plasma. The animals were then fixed on a Plexiglas stage and the observation window of the chamber was horizontally positioned under a Zeiss Axiotech microscope (Zeiss, Oberkochen, Germany) with a $100 \mathrm{~W}$ mercury lamp, which was attached to an epi-illumination filter block for blue light. The microscopic images were recorded by a charge-coupled device video camera (FK6990; Pieper, Schwerte, Germany) and transferred to a DVD system for off-line analysis. By means of $5 \mathrm{x}, 10 \mathrm{x}$ and $20 \mathrm{x}$ long-distance objectives (Zeiss) magnifications of x 115, x 230 and x 460 were achieved on a 14 inch video screen (Trinitron; Sony, Tokyo, Japan).

The microscopic images were analysed by means of the software package CapImage (Zeintl, Heidelberg, Germany). Vascularisation of the scaffolds was evaluated at a magnification of $x 460$ in 8 different microvascular regions of interest (ROIs) within the border zone and 8 different microvascular ROIs within the centre of the implants. Perfused ROIs (given in \% of all analysed ROIs) were defined as areas, which exhibited either newly developed red blood cell (RBC)-perfused microvessels or reperfused microvascular fragments. In addition, the functional microvessel density, i.e., the length of all RBCperfused blood vessels per ROI, was measured and is given in $\mathrm{cm} / \mathrm{cm}^{2}$ (Laschke et al., 2012). Finally, we assessed the diameter ( $\mathrm{d}$, given in $\mu \mathrm{m}$ ) and the centreline RBC velocity ( $\mathrm{v}$, given in $\mu \mathrm{m} / \mathrm{s}$ ) of 25 randomly selected microvessels in the centre of the implants, which already exhibited a blood
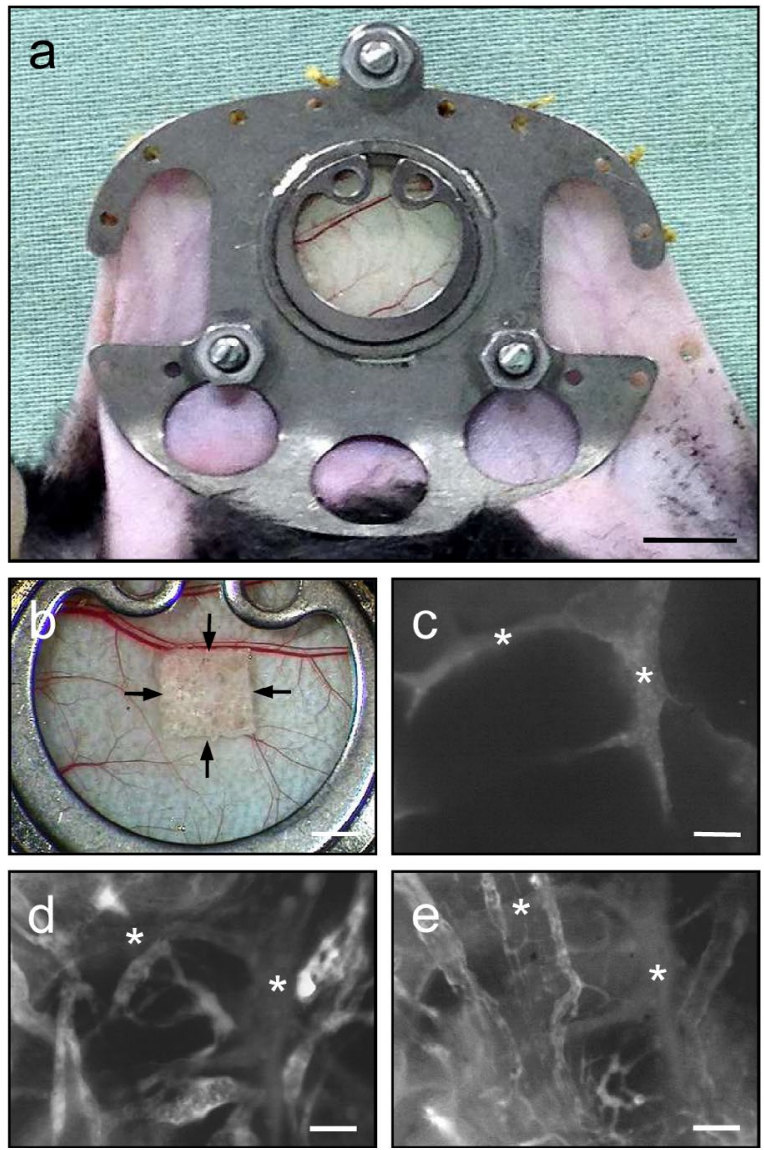

Fig. 2. (a) Dorsal skinfold chamber (chamber weight $\sim 2 \mathrm{~g}$ ) on the back of a C57BL/6 mouse. (b) Overview of the observation window of a chamber directly after implantation of a fragment-seeded polyurethane scaffold ( $\sim 3 \times 3 \times 1 \mathrm{~mm}$; borders marked by arrows). (ce) Intravital fluorescence microscopy of a central ROI of a polyurethane scaffold seeded with microvascular fragments from an adult donor mouse directly (c) as well as at day 10 (d) and 14 (e) after implantation into the dorsal skinfold chamber. The scaffold initially lacks a vascularisation (c). However, due to the rapid reassembly of the seeded microvascular fragments the centre of the implant finally exhibits a dense network of FITC-dextran-labelled blood-perfused microvessels (d, e). Blue-light epi-illumination with contrast enhancement by $5 \%$ FITC-labelled dextran 150,000 i.v. Scaffold strands (c-e, asterisks) appear grey under these imaging conditions. Scale bars: (a) $=5.6 \mathrm{~mm} ;(\mathbf{b})=1.5 \mathrm{~mm} ;(\mathbf{c}-\mathbf{e})=60 \mu \mathrm{m}$.

perfusion at the time point of analysis. The wall shear rate ( $y$, given in $1 / \mathrm{s}$ ) of these vessels was then calculated based on the Newtonian definition: $y=8 * v / d$ (Ehrmantraut et al., 2010).

\section{Experimental protocol}

Microvascular fragments from 8 adult and 8 aged mice were seeded on 16 scaffolds and implanted into the dorsal skinfold chamber of 16 C57BL/6 recipient mice $(n=8$ per group). Intravital fluorescence microscopy was performed directly after implantation and the implants' vascularisation 
was analysed at days 3, 6, 10 and 14 . At the end of the in vivo experiments, the animals were sacrificed with an overdose of the anaesthetic and the dorsal skinfold preparations were excised for further histological and immunohistochemical analyses.

In a subset of experiments, microvascular fragments from additional 4 adult mice and 4 aged mice were seeded on 8 scaffolds ( $n=4$ per group), which were transferred to a 48 -well plate filled with $550 \mu \mathrm{L}$ endothelial cell growth medium MV (PromoCell, Heidelberg, Germany) per well. To simulate in vitro hypoxic stress, the seeded scaffolds were cultivated for $18 \mathrm{~h}$ in a chamber at constant gas flow $\left(95 \% \mathrm{~N}_{2}, 5 \% \mathrm{CO}_{2}\right)$ at $37^{\circ} \mathrm{C}$. This assured an $\mathrm{O}_{2}$ saturation of $<5 \mathrm{~mm} \mathrm{Hg}$ in the culture medium, which is comparable to an $\mathrm{O}_{2}$ atmosphere of $0.5 \%$ (Oberringer et al., 2005; Purins et al., 2010). After cultivation, the concentration of vascular endothelial growth factor (VEGF) and basic fibroblast growth factor (bFGF) were analysed in culture medium supernatants by enzyme-linked immunosorbent assay (ELISA) and the scaffolds were preserved for further immunohistochemical analyses.

\section{Histology and immunohistochemistry}

Formalin-fixed specimens of cultivated scaffolds as well as dorsal skinfold preparations with scaffold implants were embedded in paraffin and cut into $2 \mu \mathrm{m}$-thick sections. Haematoxylin and eosin (HE) staining of individual sections was performed according to standard procedures.

For immunohistochemical analyses, paraffin-embedded $2 \mu \mathrm{m}$-thick sections were stained with a monoclonal rat-anti-mouse antibody against $\operatorname{CD} 31$ (1:30; dianova, Hamburg, Germany), with a polyclonal rabbit-anti-human antibody against $\alpha$-smooth muscle actin ( $\alpha$-SMA; 1:100; Abcam, Cambridge, UK), with a monoclonal mouseanti-mouse antibody against proliferating cell nuclear antigen (PCNA; 1:100, Dako, Hamburg, Germany), with a polyclonal rabbit-anti-mouse antibody against cleaved caspase-3 (Casp-3; 1:100; New England Biolabs, Frankfurt am Main, Germany) and with a polyclonal rabbit-antimouse antibody against matrix metalloproteinase-9 (MMP9; 1:25; Abcam). A goat-anti-rat IgG-Cy3 antibody (1:50; dianova $\mathrm{GmbH})$, a goat-anti-rat IgG-Alexa488 antibody (1:50; Invitrogen, Eugene, OR, USA), a goat-anti-rabbit IgG-Cy3 antibody (1:200; dianova), a goat-anti-mouse IgG-biotinylated antibody (1:200; dianova) and a goatanti-rabbit IgG-biotinylated antibody (ready-to-use; Abcam) served as secondary antibodies. The biotinylated antibodies were detected by $\mathrm{Cy} 3$-labeled-streptavidin (1:20; Invitrogen). On each section, cell nuclei were stained with Hoechst 33342 (1:500; Sigma-Aldrich) to merge the images exactly. The sections were examined using a BX60 microscope (Olympus, Hamburg, Germany). Quantitative analyses of the sections included the determination of the microvessel density (CD31-stained cells; given in $\mathrm{mm}^{-2}$ ) and the fraction of $\alpha$-SMA-positive microvessels (double staining with CD31; \%) in the border and centre of implanted scaffolds. Moreover, we assessed the fraction of PCNA-positive or Casp-3-positive endothelial cells (double staining with CD31) as well as MMP-9-positive perivascular cells (double staining with $\alpha$-SMA) of microvascular fragments within cultivated scaffolds.

\section{ELISA}

The amount of VEGF and bFGF released from cultivated scaffolds was measured in $100 \mu \mathrm{L}$ culture medium by means of ELISA kits (VEGF: R\&D Systems Europe, Abingdon, UK; bFGF: RayBiotech, Norcross, GA, USA) using recombinant murine VEGF and bFGF as standards. Each sample was assayed in duplicate. The values were corrected to blank values (culture medium without incubated scaffolds).

\section{Flow cytometric analysis of microvascular fragments}

To analyse the stem cell content of microvascular fragments from adult and aged donors, we performed additional flow cytometric analyses. For this purpose, microvascular fragments from 10 adult donor mice and 12 aged donor mice (pooled in two separate assays) were digested in Accutase $^{\circledR}$ (BioLegend, Fell, Germany) for $30 \mathrm{~min}$ into single cells. Subsequently, the cells were analysed for the expression of the mesenchymal stem cell markers monoclonal rat-anti-mouse CD117-FITC (BD Pharmingen, Heidelberg, Germany) and unlabelled monoclonal rat-antimouse CD73 (BD Pharmingen) as well as the endothelial progenitor cell marker combination monoclonal rat-antimouse Sca-1-FITC (BD Pharmingen) and monoclonal rat-anti-mouse VEGFR-2-PE (BD Pharmingen). Isotype-

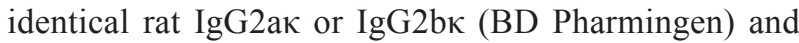
goat-anti-rat IgG-Cy3 (dianova $\mathrm{GmbH}$ ) served as controls. Flow cytometric analyses were performed by means of a FACScan (BD Pharmingen). Data were evaluated by the software package CellQuest (BD Pharmingen).

\section{Statistics}

After testing the data for normal distribution and equal variance, differences between the two groups were analysed by the unpaired Student's $t$-test. To test for time effects in the individual groups, ANOVA for repeated measures was applied. This was followed by the StudentNewman-Keuls test, including the correction of the alpha error according to Bonferroni probabilities to compensate for multiple comparisons (SigmaStat; Jandel Corporation, San Rafael, CA, USA). All values are expressed as means \pm SEM. Statistical significance was accepted for a value of $p<0.05$.

\section{Results}

\section{Vascularisation of implanted scaffolds}

After implantation into the dorsal skinfold chamber, the vascularisation of seeded scaffolds could be repetitively visualised over an observation period of 14 days by means of intravital fluorescence microscopy (Figs. 2c-e). During the first 3 days, newly formed vascular sprouts were detected in the border zones of the scaffolds as a typical early sign of the angiogenic host tissue reaction to the implants. These sprouts originated from capillary and venular segments of the host microvasculature and progressively grew towards the scaffold strands. At day 6 after implantation, we detected first blood-perfused microvessels in the scaffolds' centre. This finding proves that at this early time point the seeded microvascular 

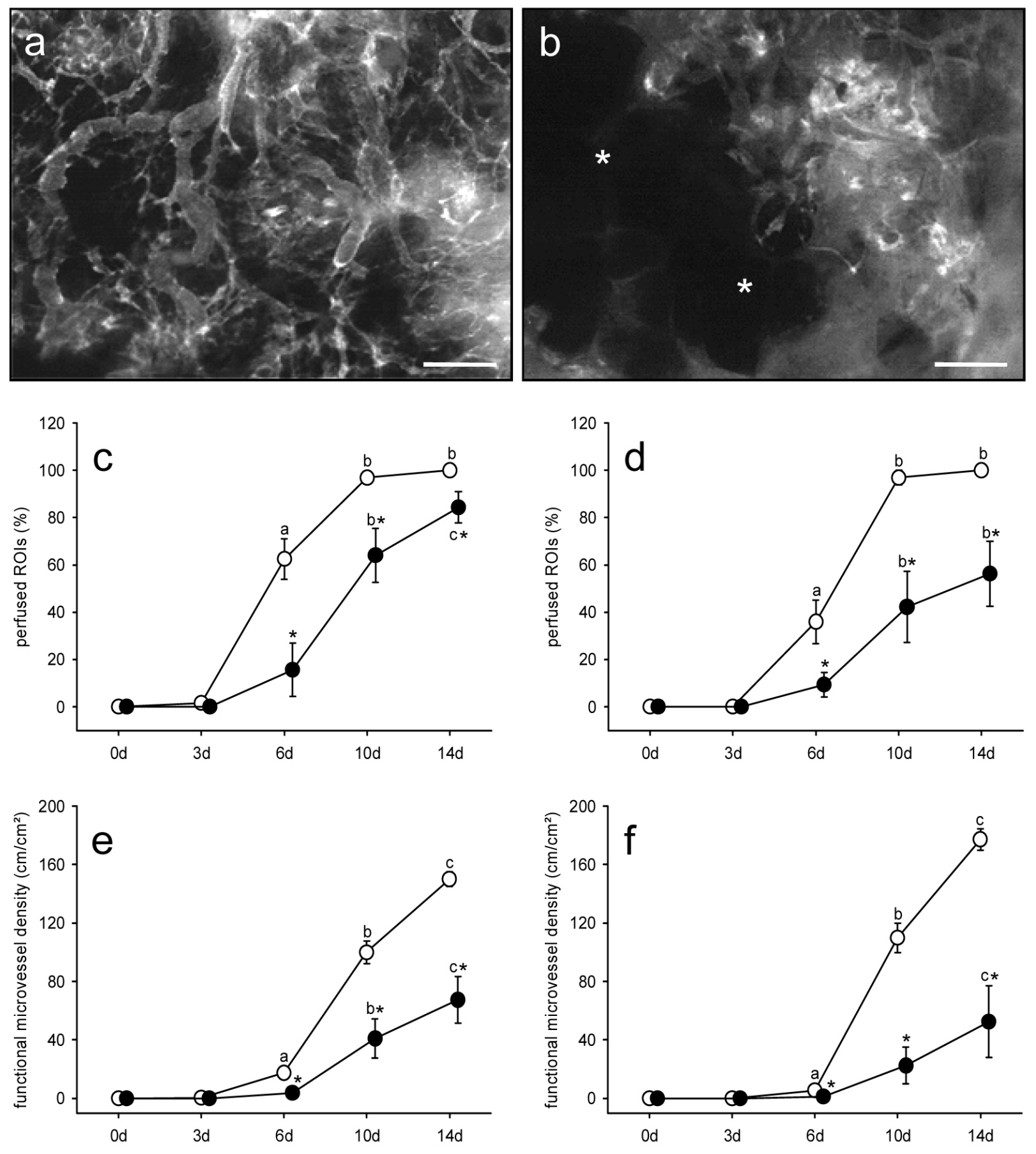

Fig. 3. (a, b) Intravital fluorescence microscopy of the centre of a polyurethane scaffold seeded with microvascular fragments from an adult (a) and an aged donor mouse (b) at day 14 after implantation into the dorsal skinfold chamber. The scaffold seeded with microvascular fragments from the adult donor contains a dense network of FITC-dextran-labelled blood-perfused microvessels (a), whereas the scaffold seeded with microvascular fragments from the aged donor still exhibits large areas lacking a blood supply (b, asterisks). Blue-light epi-illumination with contrast enhancement by $5 \%$ FITC-labelled dextran 150,000 .v. Scale bars: $320 \mu \mathrm{m}$. (c-f) Perfused ROIs (\%) (c, d) and functional microvessel density $(\mathbf{e}, \mathbf{f})$ within the border $(\mathbf{c}, \mathbf{e})$ and the centre $(\mathbf{d}, \mathbf{f})$ of polyurethane scaffolds seeded with microvascular fragments derived from adult (white circles, $n=8$ ) and aged donor mice (black circles, $n=8$ ) after implantation into dorsal skinfold chambers, as assessed by intravital fluorescence microscopy and computerassisted image analysis. Means \pm SEM. ${ }^{\mathrm{a}} p<0.05 v s .0 \mathrm{~d}$ and $3 \mathrm{~d}$ within each individual group; ${ }^{\mathrm{b}} p<0.05 v s .0 \mathrm{~d}, 3 \mathrm{~d}$ and $6 \mathrm{~d}$ within each individual group; ${ }^{\mathrm{c}} p<0.05$ vs. $0 \mathrm{~d}, 3 \mathrm{~d}, 6 \mathrm{~d}$ and $10 \mathrm{~d}$ within each individual group; ${ }^{*} p<0.05 v s$. adult donors. 
fragments had already developed interconnections to the surrounding blood vessels of the chamber tissue. Throughout the further time course of the experiment, an increasing number of microvascular fragments reassembled. Moreover, they served as the origin of new vessel sprouts, finally resulting in the formation of bloodperfused microvascular networks within the implants.

Importantly, this vascularisation process markedly differed between implanted scaffolds, which were seeded with microvascular fragments from adult and aged fat donors. In fact, the scaffolds in the group of aged donors exhibited a delayed vascularisation, as indicated by a significantly reduced number of perfused ROIs in their border and centre zones between day 6 and 14 after implantation when compared to those in the adult group (Figs. 3a-d). In addition, the newly developing microvascular networks in the group of aged donors presented also with a significantly lower functional microvessel density (Figs. 3a, b, e and f).

More detailed intravital fluorescent microscopic analyses of microhaemodynamic parameters further revealed that reperfused microvascular fragments in the scaffolds' centre of both groups exhibited comparable diameters of 50-60 $\mu \mathrm{m}$ at day 6 (Fig. 4c). During the further time course, however, diameters of microvascular fragments from adult donors progressively decreased, whereas microvascular fragments from aged donors presented with vessel wall irregularities and constant diameters until the end of the experiment (Figs. 4a-c). The decline in vessel diameters in the group of adult donor fragments was associated with an increase of the centreline RBC velocity (Fig. 4d). Accordingly, calculated values of the wall shear rate also progressively increased in this group (Fig. 4e). In contrast, blood-perfused fragments from aged donors exhibited a constant centreline RBC velocity and wall shear rate, which were markedly reduced at day 14 when compared to those of the fragments from adult donors (Figs. 4d,e).

\section{Incorporation and angioarchitecture of implanted scaffolds}

At day 14 after implantation into the dorsal skinfold chamber, we additionally analysed the scaffolds by means of histology and immunohistochemistry. In both groups, we detected a newly formed granulation tissue in the border zones and the pores of the implants. However, this granulation tissue exhibited a lower cellular density in the scaffolds seeded with microvascular fragments from aged donors (Figs. 5a,b). Moreover, it presented with a significantly reduced microvessel density in the border and centre zones of the implants when compared to the other group (Figs. 5c, d and i). Taken together, these findings indicate a deteriorated incorporation of the scaffolds
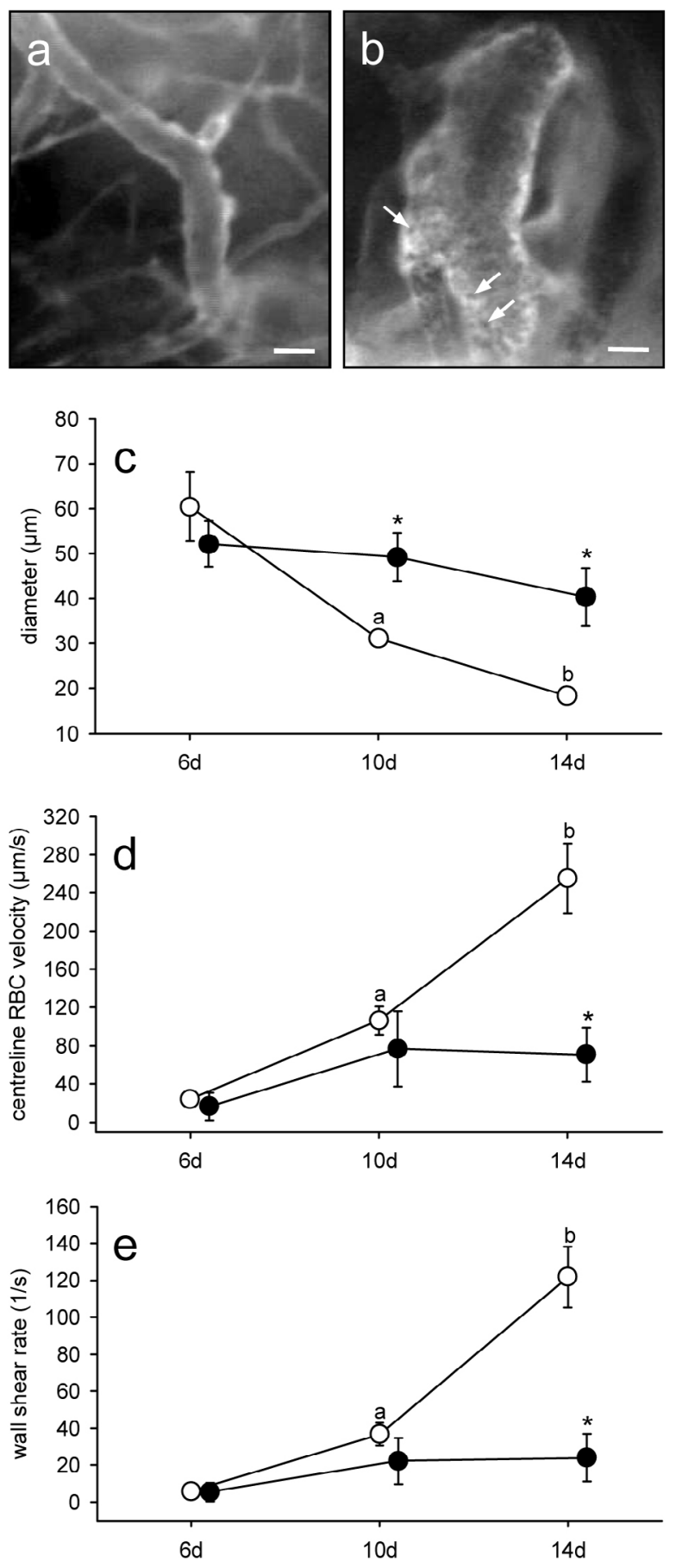

Fig. 4. (a, b) Intravital fluorescence microscopy of a typical blood-perfused microvessel in the centre of a polyurethane scaffold seeded with microvascular fragments from an adult (a) and an aged donor mouse (b) at day 14 after implantation into the dorsal skinfold chamber. Note that the microvessel in (b) exhibits a much larger diameter and a lower blood flow (indicated by the visualisation of individual black-appearing erythrocytes (arrows) in the vessel lumen) when compared to the microvessel in (a). Blue-light epi-illumination with contrast enhancement by $5 \%$ FITC-labelled dextran 150,000 i.v.. Scale bars: $30 \mu \mathrm{m}$. (c-e) Diameter $(\mu \mathrm{m})(\mathbf{c})$, centreline RBC velocity $(\mu \mathrm{m} / \mathrm{s})(\mathbf{d})$ and wall shear rate $(1 / \mathrm{s})(\mathbf{e})$ of blood-perfused microvessels in the centre of polyurethane scaffolds seeded with microvascular fragments derived from adult (white circles, $n=8$ ) and aged donor mice (black circles, $n=8$ ) after implantation into dorsal skinfold chambers, as assessed by intravital fluorescence microscopy and computer-assisted image analysis. Means \pm SEM. ${ }^{\mathrm{a}} p<0.05 v s .6 \mathrm{~d}$ within each individual group; ${ }^{\mathrm{b}} p<0.05 \mathrm{vs} .6 \mathrm{~d}$ and $10 \mathrm{~d}$ within each individual group; 

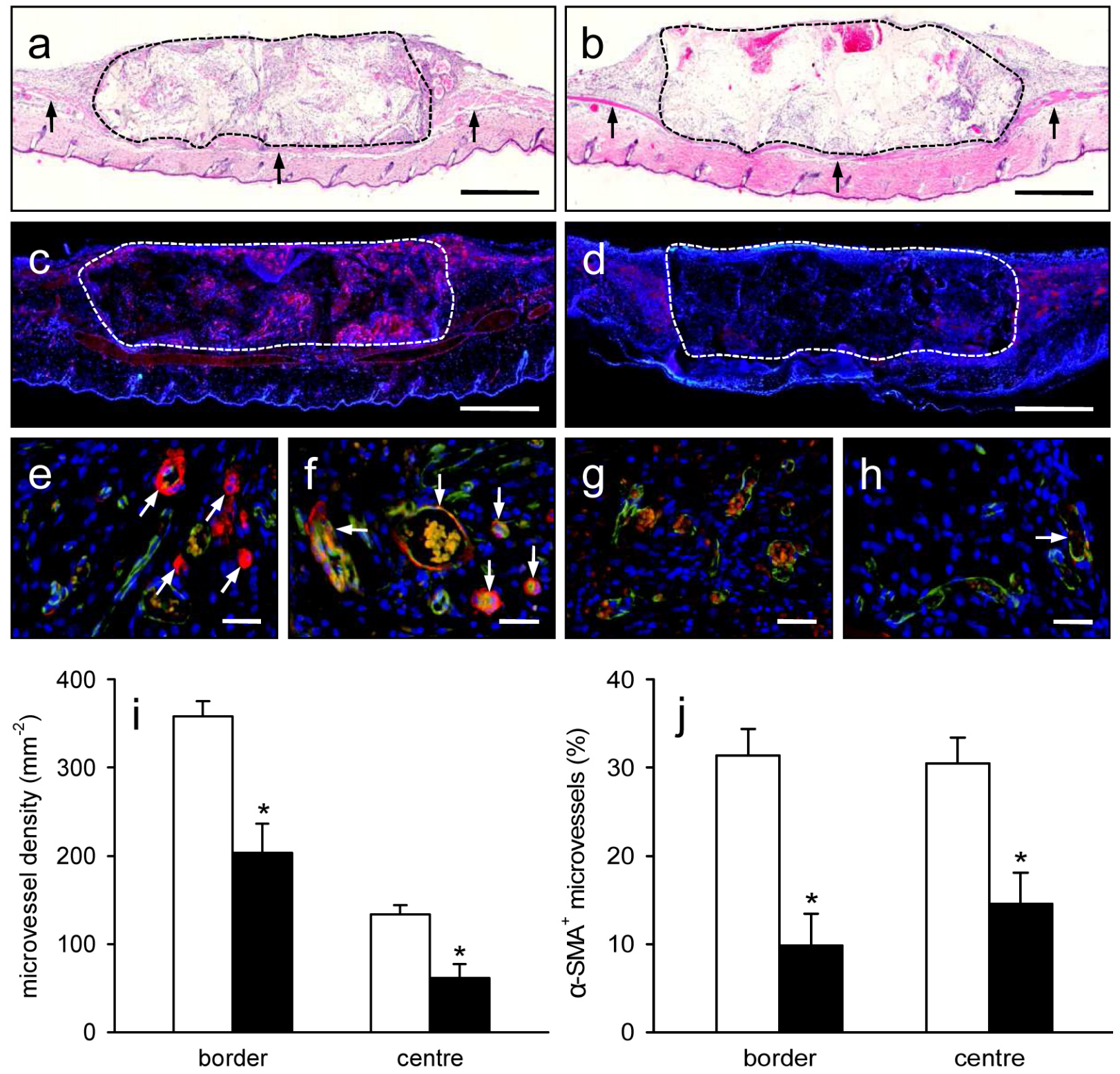

Fig. 5. (a, b) HE-stained sections of a polyurethane scaffold (borders marked by broken line) seeded with microvascular fragments from an adult (a) and an aged donor mouse (b) at day 14 after implantation onto the host striated muscle tissue (arrows) within the dorsal skinfold chamber. Note that the newly formed granulation tissue surrounding and invading the implants exhibits a lower cellular density in (b) when compared to (a). Scale bars: $650 \mu \mathrm{m}$. (c, d) Immunohistochemical detection of CD31-positive microvessels (red) in a polyurethane scaffold (borders marked by broken line) seeded with microvascular fragments from an adult (c) and an aged donor mouse (d). The sections are stained with Hoechst 33342 to identify cell nuclei. Note the markedly higher microvessel density in (c) when compared to (d). Scale bars: $650 \mu \mathrm{m}$. (e-h) Immunohistochemical detection of CD31 (green) and $\alpha$-SMA (red) in the border $(\mathbf{e}, \mathbf{g})$ and the centre $(\mathbf{f}, \mathbf{h})$ of a polyurethane scaffold seeded with microvascular fragments from an adult $(\mathbf{e}, \mathbf{f})$ and an aged donor mouse $(\mathbf{g}, \mathbf{h})$. The sections are stained with Hoechst 33342 to identify cell nuclei. Note the higher number of CD31/ $\alpha$-SMA-double positive microvessels (arrows) in (e) and (f) when compared to (g) and (h). Scale bars: $27 \mu \mathrm{m}$. (i, j) Microvessel density $\left(\mathrm{mm}^{-2}\right)$ (i) and $\alpha$-SMA-positive microvessels (\%) (j) in the border and centre of polyurethane scaffolds seeded with microvascular fragments derived from adult (white bars, $n=8$ ) and aged donor mice (black bars, $n=8$ ) at day 14 after implantation into dorsal skinfold chambers, as assessed by quantitative immunohistochemical analysis. Means \pm SEM. ${ }^{*} p<0.05 v s$. adult donors.

seeded with microvascular fragments from aged donors into the surrounding tissue.

In addition, we assessed the fraction of mature $\alpha$-SMApositive microvessels in the border and centre zones of the implants of both groups. Of interest, we found that the scaffolds seeded with microvascular fragments from aged donors contained a significantly lower number of mature microvessels when compared to scaffolds seeded with microvascular fragments from adult donors (Figs. 5e, f, g, h and $\mathrm{j}$ ). This indicates that the process of vessel maturation is markedly impaired in microvascular fragments from aged donors. 
Fig. 6. (a, b) Immunohistochemical detection of CD31 (green) and MMP-9 (red) in microvascular fragments from an adult (a) and an aged donor mouse (b) after seeding on polyurethane scaffolds and cultivation for $18 \mathrm{~h}$ under hypoxic conditions. The sections are stained with Hoechst 33342 to identify cell nuclei. Note that in contrast to (b), the microvascular fragment in (a) contains many perivascular cells, which exhibit a strong MMP-9 expression (arrows). Scale bars: $11 \mu \mathrm{m}$. (c-e) PCNA-positive endothelial cells (\%) (c), Casp-3positive endothelial cells (\%) (d) and MMP-9-positive perivascular cells $(\%)(\mathbf{e})$ in microvascular fragments derived from adult (white bars, $n=4$ ) and aged donor mice (black bars, $n=4$ ) after seeding on polyurethane scaffolds and cultivation for $18 \mathrm{~h}$ under hypoxic conditions, as assessed by quantitative immunohistochemical analysis. Means \pm SEM. ${ }^{*} p<0.05 v s$. aged donors.
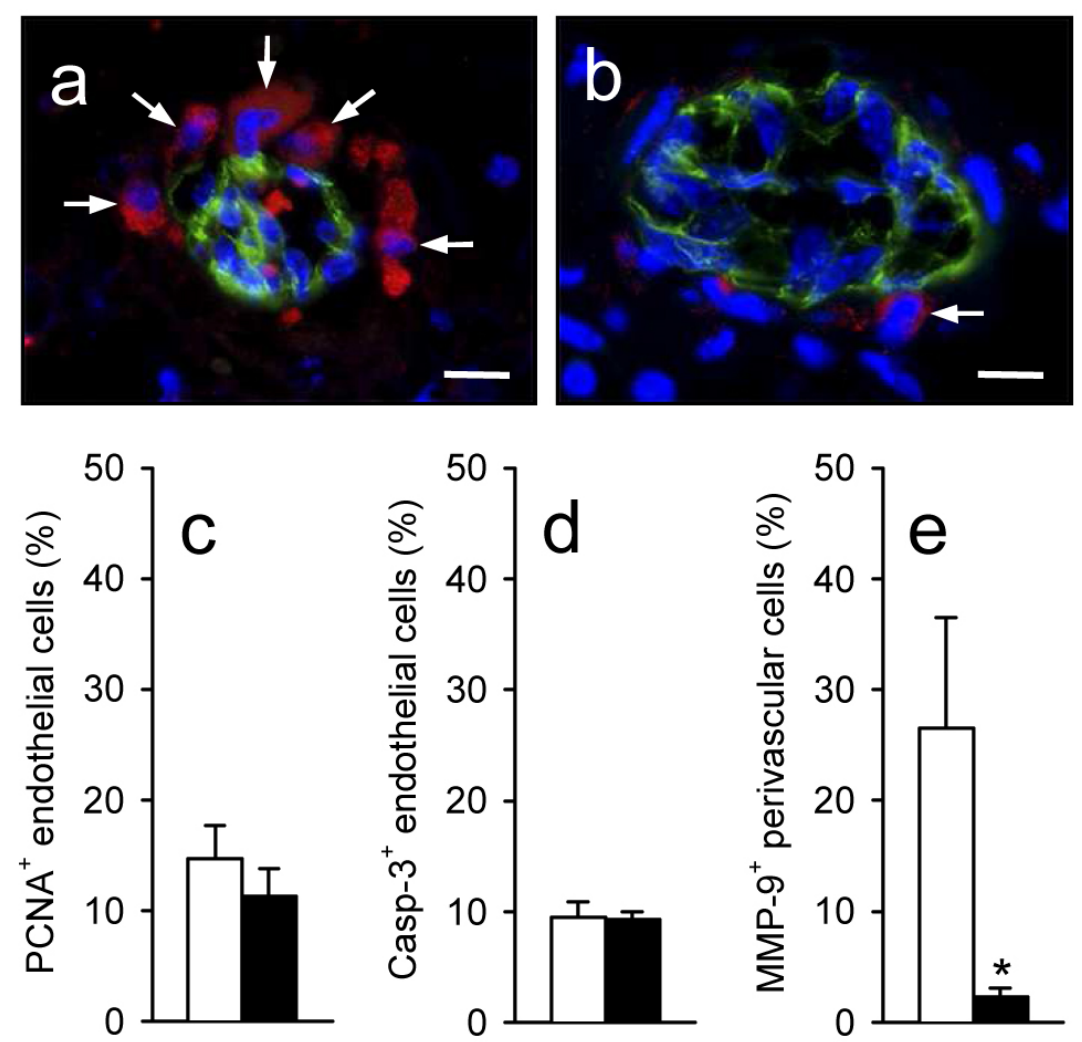

\section{Regenerative properties of hypoxia-exposed microvascular fragments}

To study the regenerative properties of microvascular fragments in a controlled in vitro setting, we cultivated fragment-seeded scaffolds for $18 \mathrm{~h}$ under hypoxic conditions, as they would also occur during the initial hours after scaffold implantation into a defect site. After this hypoxic phase, we measured the levels of angiogenic growth factors released in the culture medium. We found that VEGF and bFGF levels did not significantly differ between scaffolds seeded with microvascular fragments from adult (VEGF: $35 \pm 6 \mathrm{pg} / \mathrm{mL}$, bFGF: $541 \pm 74 \mathrm{pg}$ / $\mathrm{mL}$ ) and aged donors (VEGF: $28 \pm 4 \mathrm{pg} / \mathrm{mL}$, bFGF: $428 \pm 50 \mathrm{pg} / \mathrm{mL})$.

We next analysed the proliferative activity and survival of endothelial cells within the microvascular fragments by means of immunohistochemistry. For this purpose, the cells were stained with the endothelial marker CD31 to distinguish them from the surrounding perivascular cells. In addition, the sections were stained with antibodies against the proliferation marker PCNA and the apoptosis marker Casp-3. By this, we could demonstrate that microvascular fragments from adult and aged donors exhibited a comparable number of PCNA-positive and Casp-3-positive endothelial cells (Figs. 6c,d).

Finally, the sections were stained with an antibody against MMP-9, which is a crucial regulator of the invasive and migratory behaviour of endothelial cells and perivascular smooth muscle cells during angiogenesis and vascular remodelling (Chen et al., 2013). Of interest, MMP-9 was only expressed in a very few endothelial cells $(<1 \%)$ of microvascular fragments from adult and aged donors. However, we detected a strong MMP-9 expression in many perivascular cells of microvascular fragments from adult donors (Figs. 6a,e), which was almost completely abolished in the group of aged donors (Figs. 6b,e).

\section{Stem cell content of microvascular fragments}

To clarify, whether there is a difference in the stem cell content between microvascular fragments from adult and aged donors, we performed additional flow cytometric analyses. Freshly isolated microvascular fragments from adult donors contained $7 \pm 3 \%$ and $2 \pm 1 \%$ of cells, which expressed the mesenchymal stem cell markers CD73 and CD117, respectively. Moreover, they contained $2 \pm 0 \%$ Sca-1/VEGFR-2-positive EPCs. Of interest, the stem cell content of microvascular fragments from aged donors did not differ from these values (CD73: $6 \pm 3 \%$, CD117: $1 \pm 0 \%$, Sca-1/VEGFR-2: $2 \pm 0 \%$ ).

\section{Discussion}

In recent years, a growing number of studies indicate that the transplantation of adipose tissue-derived microvascular fragments represents a highly promising vascularisation strategy in the field of regenerative medicine and tissue engineering (Shepherd et al., 2007; Hiscox et al., 2008; Laschke et al., 2012; Pilia et al., 2014). In fact, this approach is of particular clinical relevance in that microvascular fragments may be rapidly isolated from fat tissue samples of a patient and directly transferred back into a defect site in an intra-operative one-step procedure. However, as for any other cell-based autologous approach (Stolzing et al., 2008; Steinert et al., 2012), the regenerative potential of these vessel segments may be 
crucially determined by distinct patient characteristics. In line with this view, our novel experimental data indicate that microvascular fragments from aged donors exhibit an impaired vascularisation capacity.

Although the use of microvascular fragments circumvents the need for a time-consuming de novo vessel formation within tissue constructs, the reassembly of these fragments into new microvascular networks and their interconnection with the surrounding host microvasculature also involves distinct steps of angiogenesis and vascular remodelling (Chang et al., 2010; Nunes et al., 2010). By now, there is no doubt that these processes are markedly impaired in the elderly, which likely contributes to the increased prevalence of cardiovascular diseases and their sequelae in this patient population (Lähteenvuo and Rosenzweig, 2012). Accumulating evidence, however, indicates that there may be major discrepancies between the molecular and cellular mechanisms, which impair blood vessel formation under different conditions. For instance, angiogenesis is affected in the elderly during wound healing or limb ischemia (Rivard et al., 1999; Swift et al., 1999) but does not show any alterations in response to exercise training (Rossiter et al., 2005; Gavin et al., 2007). Accordingly, we focused in our study on several mechanisms, which may have specifically contributed to the impaired vascularisation capacity of microvascular fragments from aged donors in the present setting.

After implantation into the dorsal skinfold chamber, the vascularisation of fragment-seeded scaffolds was analysed by means of intravital fluorescence microscopy. Importantly, this approach did not only enable the repetitive in vivo visualisation of network formation within the implants but also allowed for the assessment of microhaemodynamic parameters. By this, we could demonstrate that the reperfusion of microvascular fragments from adult donors was associated with a progressive decrease of the microvascular diameter and an increase of the centreline RBC velocity. This observation is typical for the maturation and vascular remodelling of developing microvascular networks within different types of tissue grafts during the initial days after transplantation (Menger et al., 1989; Vollmar et al., 2001; Laschke et al., 2006b). In consequence, calculated values of wall shear rates of individual microvessels increased continuously over time. Considering the fact that shear stress-induced mechanical forces promote angiogenesis (Brown and Hudlika, 2003; Resnick et al., 2003), this mechanism may have markedly contributed to the accelerated formation of dense microvascular networks in the implants' centre of this group.

In contrast, microvessels in scaffolds seeded with microvascular fragments derived from aged donors exhibited constantly elevated diameters with vessel wall irregularities and low centreline $\mathrm{RBC}$ velocities throughout the observation period. This indicates a disturbed maturation of the newly developing microvasculature. The maturation of microvessels is characterised by the recruitment of perivascular pericytes or smooth muscle cells, which stabilise the vessel wall, resulting in a regular shape of the vessel and a reduction of the vessel calibre (Hellström et al., 2001). In addition, it has been shown that immature blood vessels finally undergo regression (Papetti and Herman, 2002). In line with these findings, we detected a significantly reduced fraction of CD31/ $\alpha$-SMA-positive microvessels and a markedly lower microvessel density in the border and centre zones of scaffolds seeded with microvascular fragments derived from aged donors.

For a more detailed analysis of the regenerative properties of microvascular fragments, we next chose a controlled in vitro setting, mimicking the challenging environment within an implanted scaffold during the early avascular phase after implantation into a defect site. For this purpose, we cultivated fragment-seeded scaffolds for $18 \mathrm{~h}$ under hypoxic conditions. It is well known that hypoxia is one of the strongest driving forces for the production and release of angiogenic growth factors (Choi et al., 2003) and that the underlying mechanisms are impaired in the elderly (Lähteenvuo and Rosenzweig, 2012). In our experiment, however, levels of released VEGF and bFGF in the culture medium did not markedly differ between scaffolds seeded with microvascular fragments from adult and aged donors. In contrast to other studies (Wang et al. 2004; Wang et al., 2014), we also did not detect any age-dependent differences in the number of proliferating and apoptotic endothelial cells. These results indicate that the impaired vascularisation capacity of microvascular fragments from aged donors is not primarily caused by a deteriorated angiogenic activation of the endothelium or endothelial cell survival.

We next analysed the expression of MMP-9 in the fragments. This proteolytic endopeptidase is crucially involved in vascular remodelling by regulating the degradation of extracellular matrix components and the recruitment of pericytes (Chantrain et al., 2006; Chen et al., 2013). Of interest, we detected a strong MMP-9 expression in many perivascular cells of microvascular fragments from adult donors, which was almost completely abolished in the group of aged donors. This observation is in line with our in vivo finding that the process of network maturation is impaired in implanted scaffolds seeded with microvascular fragments derived from aged donors. In addition, it has recently been shown that engrafted vascular networks typically wrap around existing host vessels and express high levels of MMP-9 and MMP-14. This results in the disruption of the underlying host vessel, thereby diverting blood flow into the nascent, implanted network (Cheng et al., 2011). Taking this novel mechanism of 'wrappingand-tapping anastomosis' into account in the present study, down-regulation of perivascular MMP-9 expression may have markedly affected the rapid reassembly of fragments derived from aged donors into functional microvascular networks and their interconnection with the surrounding host microvasculature.

Previous studies indicate that microvascular fragments are a source of several types of regenerative stem cells (Laschke et al., 2012; McDaniel et al., 2014). Accordingly, we finally analysed the stem cell content of microvascular fragments from young and old donor mice by means of flow cytometry. We found that both fragment types exhibited a comparable number of CD73- and CD117positive mesenchymal stem cells as well as Sca-1/VEGFR2-positive EPCs. This, however, does not necessarily 
mean that the regenerative potential of these cells is also comparable in both groups. In fact, recent reports indicate that aging alters the proliferation, differentiation and angiogenic activity of adipose-derived mesenchymal stem cells (Alt et al., 2012; Efimenko et al., 2014). Accordingly, further studies are needed to clarify whether this aging effect contributes to the herein observed impaired vascularisation capacity of microvascular fragments.

\section{Conclusions}

The present study demonstrates that network formation, onset of blood perfusion and vascular remodelling are markedly affected in implanted scaffolds seeded with adipose tissue-derived microvascular fragments from aged donors. In light of the fact that elderly patients will be the major target population of regenerative approaches in the future, following studies should therefore focus on novel strategies to stimulate the vascularisation capacity of microvascular fragments for different tissue engineering applications

\section{Acknowledgements}

This work was supported by the Large Bone Defect Healing Program of AO Foundation. We are grateful for the excellent technical assistance of Janine Becker, Ruth M. Nickels, Martin Oberringer and Julia Parakenings.

\section{References}

Alt EU, Senst C, Murthy SN, Slakey DP, Dupin CL, Chaffin AE, Kadowitz PJ, Izadpanah R (2012) Aging alters tissue resident mesenchymal stem cell properties. Stem Cell Res 8: 215-225.

Brown MD, Hudlicka O (2003) Modulation of physiological angiogenesis in skeletal muscle by mechanical forces: involvement of VEGF and metalloproteinases. Angiogenesis 6: 1-14.

Chang CC, Nunes SS, Sibole SC, Krishnan L, Williams SK, Weiss JA, Hoying JB (2010) Angiogenesis in a microvascular construct for transplantation depends on the method of chamber circulation. Tissue Eng Part A 16: 795-805.

Chantrain CF, Henriet P, Jodele S, Emonard H, Feron O, Courtoy PJ, DeClerck YA, Marbaix E (2006) Mechanisms of pericyte recruitment in tumour angiogenesis: a new role for metalloproteinases. Eur J Cancer 42: 310-318.

Chen Q, Jin M, Yang F, Zhu J, Xiao Q, Zhang L (2013) Matrix metalloproteinases: inflammatory regulators of cell behaviors in vascular formation and remodeling. Mediators Inflamm 2013: 928315.

Cheng G, Liao S, Kit Wong H, Lacorre DA, di Tomaso E, Au P, Fukumura D, Jain RK, Munn LL (2011) Engineered blood vessel networks connect to host vasculature via wrapping-and-tapping anastomosis. Blood 118: $4740-4749$.
Choi KS, Bae MK, Jeong JW, Moon HE, Kim KW (2003) Hypoxia-induced angiogenesis during carcinogenesis. J Biochem Mol Biol 36: 120-127.

Choudhery MS, Badowski M, Muise A, Pierce J, Harris DT (2014) Donor age negatively impacts adipose tissue-derived mesenchymal stem cell expansion and differentiation. J Transl Med 12: 8.

Duttenhoefer F, Lara de Freitas R, Meury T, Loibl M, Benneker LM, Richards RG, Alini M, Verrier S (2013) 3D scaffolds co-seeded with human endothelial progenitor and mesenchymal stem cells: evidence of prevascularisation within 7 days. Eur Cell Mater 26: 49-64.

Efimenko A, Dzhoyashvili N, Kalinina N, Kochegura T, Akchurin R, Tkachuk V, Parfyonova Y (2014) Adiposederived mesenchymal stromal cells from aged patients with coronary artery disease keep mesenchymal stromal cell properties but exhibit characteristics of aging and have impaired angiogenic potential. Stem Cells Transl Med 3: 32-41.

Ehrmantraut S, Laschke MW, Merkel D, Scheuer C, Willnecker V, Meyer-Lindenberg A, Menger MD, Naumann A (2010) Perioperative steroid administration inhibits angiogenic host tissue response to porous polyethylene (Medpor) implants. Eur Cell Mater 19: 107116.

Gavin TP, Ruster RS, Carrithers JA, Zwetsloot KA, Kraus RM, Evans CA, Knapp DJ, Drew JL, McCartney JS, Garry JP, Hickner RC (2007) No difference in the skeletal muscle angiogenic response to aerobic exercise training between young and aged men. J Physiol 585: 231-239.

Hellström M, Gerhardt H, Kalén M, Li X, Eriksson U, Wolburg H, Betsholtz C (2001) Lack of pericytes leads to endothelial hyperplasia and abnormal vascular morphogenesis. J Cell Biol 153: 543-553.

Hiscox AM, Stone AL, Limesand S, Hoying JB, Williams SK (2008) An islet-stabilizing implant constructed using a preformed vasculature. Tissue Eng Part A 14: 433440 .

Hoying JB, Boswell CA, Williams SK (1996) Angiogenic potential of microvessel fragments established in three-dimensional collagen gels. In Vitro Cell Dev Biol Anim 32: 409-419.

Lähteenvuo J, Rosenzweig A (2012) Effects of aging on angiogenesis. Circ Res 110: 1252-1264.

Laschke MW, Harder Y, Amon M, Martin I, Farhadi J, Ring A, Torio-Padron N, Schramm R, Rücker M, Junker D, Häufel JM, Carvalho C, Heberer M, Germann G, Vollmar B, Menger MD (2006a) Angiogenesis in tissue engineering: breathing life into constructed tissue substitutes. Tissue Eng 12: 2093-2104.

Laschke MW, Elitzsch A, Vollmar B, Vajkoczy P, Menger MD (2006b) Combined inhibition of vascular endothelial growth factor (VEGF), fibroblast growth factor and platelet-derived growth factor, but not inhibition of VEGF alone, effectively suppresses angiogenesis and vessel maturation in endometriotic lesions. Hum Reprod 21: 262-268.

Laschke MW, Rücker M, Jensen G, Carvalho C, Mülhaupt R, Gellrich NC, Menger MD (2008) Improvement of vascularization of PLGA scaffolds by 
inosculation of in situ-preformed functional blood vessels with the host microvasculature. Ann Surg 248: 939-948.

Laschke MW, Vollmar B, Menger MD (2009) Inosculation: connecting the life-sustaining pipelines. Tissue Eng Part B Rev 15: 455-465.

Laschke MW, Strohe A, Menger MD, Alini M, Eglin D (2010a) In vitro and in vivo evaluation of a novel nanosize hydroxyapatite particles/poly(ester-urethane) composite scaffold for bone tissue engineering. Acta Biomater 6: 2020-2027.

Laschke MW, Mussawy H, Schuler S, Eglin D, Alini M, Menger MD (2010b) Promoting external inosculation of prevascularised tissue constructs by pre-cultivation in an angiogenic extracellular matrix. Eur Cell Mater 20: 356-366.

Laschke MW, Mussawy H, Schuler S, Kazakov A, Rücker M, Eglin D, Alini M, Menger MD (2011a) Shortterm cultivation of in situ prevascularized tissue constructs accelerates inosculation of their preformed microvascular networks after implantation into the host tissue. Tissue Eng Part A 17: 841-853.

Laschke MW, Vollmar B, Menger MD (2011b) The dorsal skinfold chamber: window into the dynamic interaction of biomaterials with their surrounding host tissue. Eur Cell Mater 22: 147-164.

Laschke MW, Menger MD (2012) Vascularization in tissue engineering: angiogenesis versus inosculation. Eur Surg Res 48: 85-92.

Laschke MW, Kleer S, Scheuer C, Schuler S, Garcia P, Eglin D, Alini M, Menger MD (2012) Vascularisation of porous scaffolds is improved by incorporation of adipose tissue-derived microvascular fragments. Eur Cell Mater 24: 266-277.

Laschke MW, Schank TE, Scheuer C, Kleer S, Schuler S, Metzger W, Eglin D, Alini M, Menger MD (2013) Threedimensional spheroids of adipose-derived mesenchymal stem cells are potent initiators of blood vessel formation in porous polyurethane scaffolds. Acta Biomater 9: 68766884.

McDaniel JS, Pilia M, Ward CL, Pollot BE, Rathbone CR (2014) Characterization and multilineage potential of cells derived from isolated microvascular fragments. J Surg Res, in press

Menger MD, Jaeger S, Walter P, Feifel G, Hammersen F, Messmer K (1989) Angiogenesis and hemodynamics of microvasculature of transplanted islets of Langerhans. Diabetes 38 Suppl 1: 199-201.

Muscari C, Giordano E, Bonafè F, Govoni M, Guarnieri C (2014) Strategies affording prevascularized cell-based constructs for myocardial tissue engineering. Stem Cells Int 2014: 434169.

Novosel EC, Kleinhans C, Kluger PJ (2011) Vascularization is the key challenge in tissue engineering. Adv Drug Deliv Rev 63: 300-311.

Nunes SS, Greer KA, Stiening CM, Chen HY, Kidd KR, Schwartz MA, Sullivan CJ, Rekapally H, Hoying JB (2010) Implanted microvessels progress through distinct neovascularization phenotypes. Microvasc Res 79: 10-20.

Oberringer M, Jennewein M, Motsch SE, Pohlemann T, Seekamp A (2005) Different cell cycle responses of wound healing protagonists to transient in vitro hypoxia. Histochem Cell Biol 123: 595-603.

Orr AW, Elzie CA, Kucik DF, Murphy-Ullrich JE (2003) Thrombospondin signaling through the calreticulin/ LDL receptor-related protein co-complex stimulates random and directed cell migration. J Cell Sci 116: $2917-$ 2927.

Papetti M, Herman IM (2002) Mechanisms of normal and tumor-derived angiogenesis. Am J Physiol Cell Physiol 282: C947-970.

Pilia M, McDaniel JS, Guda T, Chen XK, Rhoads RP, Allen RE, Corona BT, Rathbone CR (2014) Transplantation and perfusion of microvascular fragments in a rodent model of volumetric muscle loss injury. Eur Cell Mater 28: 11-24.

Purins K, Enblad P, Sandhagen B, Lewén A (2010) Brain tissue oxygen monitoring: a study of in vitro accuracy and stability of Neurovent-PTO and Licox sensors. Acta Neurochir (Wien) 152: 681-688.

Resnick N, Yahav H, Shay-Salit A, Shushy M, Schubert S, Zilberman LC, Wofovitz E (2003) Fluid shear stress and the vascular endothelium: for better and for worse. Prog Biophys Mol Biol 81: 177-199.

Rivron NC, Liu J J, Rouwkema J, de Boer J, van Blitterswijk CA (2008) Engineering vascularised tissues in vitro. Eur Cell Mater 15: 27-40.

Rivard A, Fabre JE, Silver M, Chen D, Murohara T, Kearney M, Magner M, Asahara T, Isner JM (1999) Agedependent impairment of angiogenesis. Circulation 99: 111-120.

Rossiter HB, Howlett RA, Holcombe HH, Entin PL, Wagner HE, Wagner PD (2005) Age is no barrier to muscle structural, biochemical and angiogenic adaptations to training up to 24 months in female rats. J Physiol 565: 993-1005.

Shepherd BR, Chen HY, Smith CM, Gruionu G, Williams SK, Hoying JB (2004) Rapid perfusion and network remodeling in a microvascular construct after implantation. Arterioscler Thromb Vasc Biol 24: 898-904.

Shepherd BR, Hoying JB, Williams SK (2007) Microvascular transplantation after acute myocardial infarction. Tissue Eng 13: 2871-2879

Stegemann JP, Verrier S, Gebhard F, Laschke MW, Martin I, Simpson H, Miclau T (2014) Cell therapy for bone repair: narrowing the gap between vision and practice. Eur Cell Mater 27: 1-4.

Steinert AF, Rackwitz L, Gilbert F, Nöth U, Tuan RS (2012) Concise review: the clinical application of mesenchymal stem cells for musculoskeletal regeneration: current status and perspectives. Stem Cells Transl Med 1: 237-247.

Stolzing A, Jones E, McGonagle D, Scutt A (2008) Age-related changes in human bone marrow-derived mesenchymal stem cells: consequences for cell therapies. Mech Ageing Dev 129: 163-173.

Swift ME, Kleinman HK, DiPietro LA (1999) Impaired wound repair and delayed angiogenesis in aged mice. Lab Invest 79: 1479-1487.

Vollmar B, Laschke MW, Rohan R, Koenig J, Menger $\mathrm{MD}$ (2001) In vivo imaging of physiological angiogenesis from immature to preovulatory ovarian follicles. Am J Pathol 159: 1661-1670. 
Wang H, Keiser JA, Olszewski B, Rosebury W, Robertson A, Kovesdi I, Gordon D (2004) Delayed angiogenesis in aging rats and therapeutic effect of adenoviral gene transfer of VEGF. Int J Mol Med 13: 581587.

Wang H, Listrat A, Meunier B, Gueugneau M, CoudyGandilhon C, Combaret L, Taillandier D, Polge C, Attaix D, Lethias C, Lee K, Goh KL, Béchet D (2014) Apoptosis in capillary endothelial cells in ageing skeletal muscle. Aging Cell 13: 254-262.

Zarem HA (1969) The microcirculatory events within full-thickness skin allografts (homografts) in mice. Surgery 66: 392-397.

\section{Discussion with Reviewers}

M. Ioibl: You clearly demonstrated a difference regarding the weight of adult and aged C57BL/6 mice. Could you imagine a diet-induced obesity in some of the animals with resulting cardiovascular disease? Please comment!

Authors: The measured difference in weight of adult and aged C57BL/6 mice in the present study was physiological and not induced by the standard diet. However, it is possible to induce obesity in these animals by means of a specific fat diet. This could be an interesting approach to analyse in future studies the effects of cardiovascular disease on the vascularisation potential of isolated microvascular fragments.

M. Ioibl: You gathered a large body of evidence about the impaired vascularisation. The question if it clinically "matters" is not addressed in this study. Could you speculate on the clinical impact for tissue defect healing in vivo?

Authors: As already mentioned in our article, elderly patients will be the major target population of regenerative approaches in the future. Our findings indicate that the vascularisation potential of microvascular fragments may be impaired in these patients, which may markedly affect the clinical success of tissue defect healing when using this vascularisation strategy for implanted tissue constructs. Therefore, we suggest that future studies should focus on the establishment of approaches, which may help to improve the vascularisation potential of microvascular fragments in elderly patients.

M. Ioibl: Stem cell origin is crucial for various tissueengineering purposes. Have you looked at the differentiation capacity of the contained stem cells in the applied microvascular fragments? For the regeneration of which tissues do you specifically recommend microvascular fragments?

Authors: In the present study, we did not analyse the differentiation capacity of the stem cells inside the isolated microvascular fragments. However, previous studies have already shown that aging alters the differentiation of adipose-derived mesenchymal stem cells (Alt et al., 2012; Efimenko et al., 2014, text references).

We feel that the use of microvascular fragments as vascularisation units is not restricted to a specific tissue. In fact, microvascular fragments may be applied whenever a rapid and sufficient scaffold vascularisation is needed. For this purpose, microvascular fragments may also be combined with tissue-specific cells, such as keratinocytes for the generation of skin or osteoblasts for the generation of bone.

H.-G. Machens: What chance do you see to upscale the size of the matrix used in this model?

Authors: In the present study we used porous nano-size hydroxyapatite particles/poly(ester-urethane) composite scaffolds, which were fabricated by a salt leaching-phase inverse technique (Laschke et al., 2010a, text reference). The original size of these scaffolds during the production process was $2 \times 9 \mathrm{~cm}$. For the dorsal skinfold chamber experiments, the material had to be sliced into $3 \times 3 \times 1 \mathrm{~mm}$ samples by means of water jet cutting and a laser ablation technique. Accordingly, upscaling of the scaffolds for other applications is not a problem.

H.-G. Machens: Would this seeding technique be applicable in matrices for both dermal and bone replacement?

Authors: Our seeding technique is applicable for a broad range of different scaffolds as long as they exhibit interconnected pores, which allow a dynamic flow of seeding suspensions through the material. 